\title{
Linfangiectasia renal: achado incidental em tomografia computadorizada multicorte e revisão da literatura*
}

\author{
Renal lymphangiectasia: incidental finding at multislice computed tomography and literature review
}

\author{
Rodrigo Abdalla de Vasconcelos ${ }^{1}$, Emanuelle Santiago Pereira ${ }^{2}$, Tufik Bauab Junior ${ }^{3}$, Rodolfo \\ Silva Valente ${ }^{4}$
}

\begin{abstract}
Resumo Linfangiectasia renal é uma condição rara, caracterizada por coleções parapiélicas e perirrenais, que pode progredir de assintomática para insuficiência renal crônica. É apresentado um caso de achado incidental em tomografia computadorizada de linfangiectasia renal bilateral em paciente assintomático, com descrição dos principais achados à luz dos métodos de imagem e ampla revisão da literatura.
\end{abstract}

Unitermos: Linfangiectasia renal; Linfangiomatose renal; Malformação; Sistema linfático; Rim; Tomografia computadorizada.

Abstract Renal lymphangiectasia is a rare condition characterized by parapyelic and perirenal fluid collections, which may progress from asymptomatic condition to chronic renal failure. The present report describes a case of incidental computed tomography finding of bilateral lymphangiectasia in an asymptomatic patient, as well as the main imaging findings with a comprehensive literature review.

Keywords: Renal lymphangiectasia; Renal lymphangiomatosis; Malformation; Lymphatic system; Kidney; Computed tomography.

Vasconcelos RA, Pereira ES, Bauab Jr T, Valente RS. Linfangiectasia renal: achado incidental em tomografia computadorizada multicorte e revisão da literatura. Radiol Bras. 2012 Mai/Jun;45(3):178-180.

\section{INTRODUÇÃO}

Linfangiectasia renal é uma rara desordem benigna do sistema linfático dos rins, geralmente descoberta incidentalmente. Apresentamos um caso de achado incidental de linfangiectasia renal bilateral em tomografia computadorizada, com revisão da literatura e descrição dos principais achados através dos métodos de imagem.

\section{RELATO DO CASO}

Paciente do sexo feminino, 65 anos de idade, procurou o serviço de radiologia do

* Trabalho realizado no Hospital de Base do Distrito Federal (HBDF), Brasília, DF, Brasil.

1. Médico Radiologista do Ecocenter - Diagnósticos Médicos por Imagem, do Hospital de Base do Distrito Federal (HBDF) e do Hospital Universitário de Brasília (HUB), Brasília, DF, Brasil.

2. Médica Residente de Radiologia do Hospital de Base do Distrito Federal (HBDF), Brasília, DF, Brasil.

3. Médico Radiologista do Instituto de Radiodiagnóstico Rio Preto - Ultra-Xe do Hospital de Base de Faculdade de Medicina de São José do Rio Preto (Famerp), São José do Rio Preto, SP Brasil.

4. Médico Radiologista do Hospital Universitário de Brasília (HUB), Brasília, DF, Brasil.

Endereço para correspondência: Dr. Rodrigo Abdalla de Vasconcelos. SEPS 709/909 Lote A - Centro Médico Júlio Adnet, Bloco B, sala 406. Brasília, DF, Brasil, 70390-095. E-mail: ecocenterdf@gmail.com

Recebido para publicação em 14/9/2011. Aceito, após revisão, em 2/12/2011.
Hospital das Forças Armadas, Brasília, DF, com queixa de dispneia. Em sua história clínica não havia relato de outros sintomas, comorbidades ou história familiar significativa. O exame físico era normal.

Foi realizada tomografia computadorizada do tórax, em que foram evidenciadas nos cortes abdominais, incidentalmente, lesões císticas perinéfricas e parapiélicas bilateralmente, poupando o parênquima renal, de aspecto multiloculado e densidades variando de 7 a $20 \mathrm{UH}$ (média de $11 \mathrm{UH}$ ), sem captação de contraste nas fases obtidas (corticomedular e excretora). Os cistos parapiélicos determinavam discreta distorção dos sistemas pielocalicinais, sem sinais obstrutivos (Figuras 1 a 3). Assim, o diagnóstico por imagem caracterizou linfangiectasia renal.

\section{DISCUSSÃO}

Linfangiectasia é uma rara malformação benigna envolvendo o sistema linfático dos rins $^{(\mathbf{1})}$

A origem desta desordem ainda é controversa, mas acredita-se que a fisiopatogênese decorra de falha do sistema linfático regional em desenvolver comunicação com o tecido linfático sistêmico ${ }^{(\mathbf{1 - 3})}$.

A condição é encontrada em ambos os sexos, em qualquer idade ${ }^{(2,3)}$. Na grande maioria dos casos publicados o processo ocorre em ambos os rins, geralmente descoberto de forma incidental ou em pacientes que se apresentam com massa abdominal palpável e/ou dor nos flancos ${ }^{(\mathbf{4})}$.

O diagnóstico pode ser feito de forma confiável apenas pelos métodos de imagem na grande maioria das vezes, já que seus achados são bastante característicos ${ }^{(2,3)}$.

$\mathrm{Na}$ ultrassonografia essas lesões são visualizadas como massas anecoicas septadas com reforço acústico posterior, podendo haver finos ecos em suspensão ou débris em caso de hemorragia ${ }^{(\mathbf{1}, 3,5)}$. Adicionalmente, pode haver hiperecogenicidade de ambos os rins, com perda da diferenciação corticomedular $^{(\mathbf{1 , 4 , 6})}$.

A tomografia computadorizada identifica massas císticas uni ou multiloculadas de paredes finas, preenchidas por material com atenuação de líquido, sem realce pelo meio de contraste, podendo comprimir o córtex, expandir o seio renal e distorcer o sistema calicinal ${ }^{(\mathbf{1}, 2,5,6)}$. Em casos de hemor- 


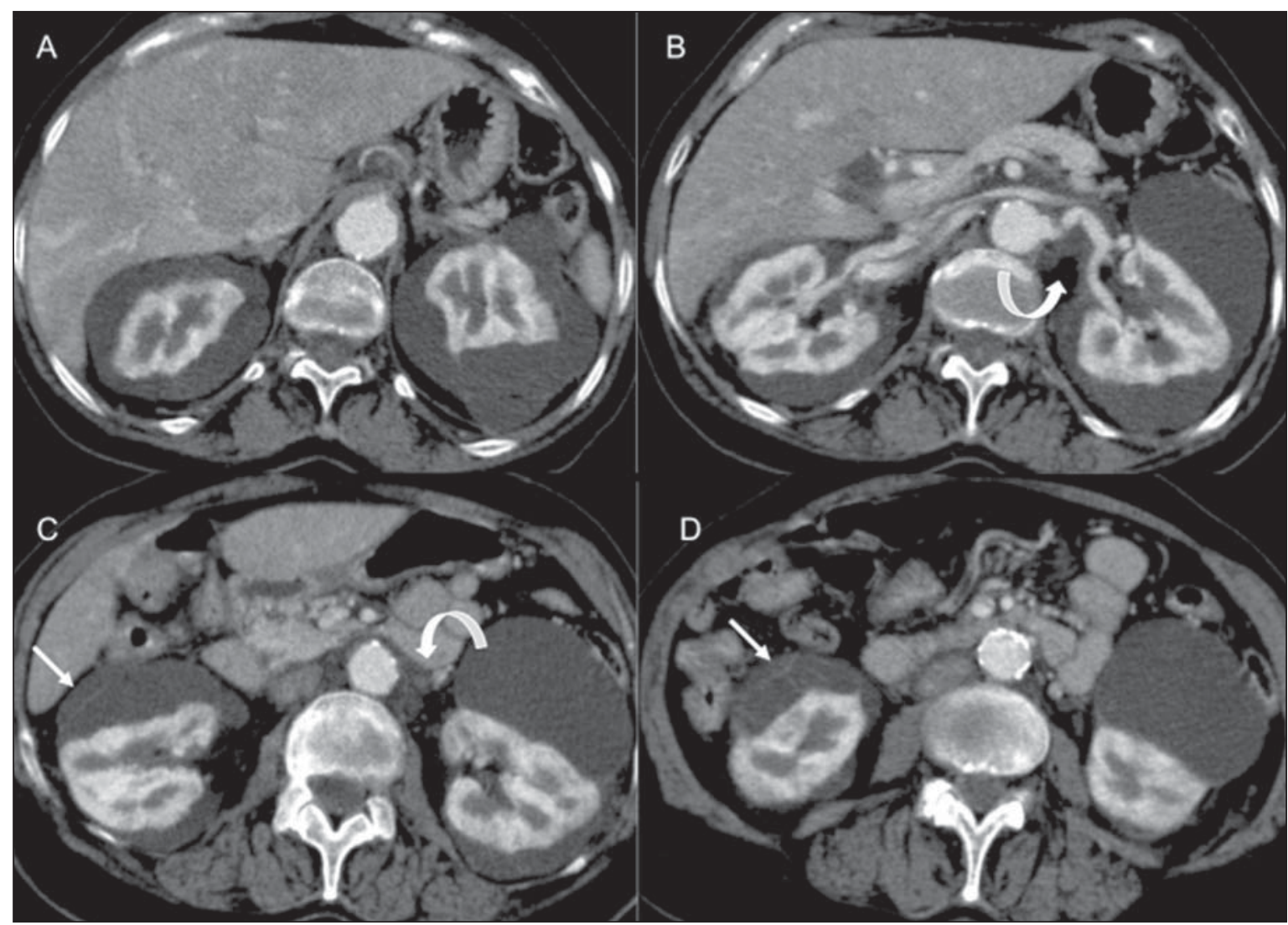

Figura 1. Imagens axiais volumétricas após meio de contraste iodado intravenoso demonstrando volumosas imagens císticas perinéfricas, com finas septações internas (setas retas em $\mathbf{C}$ e D) e extensão para o hilo renal e espaço periaórtico (setas curvas em $\mathbf{B}$ e C).

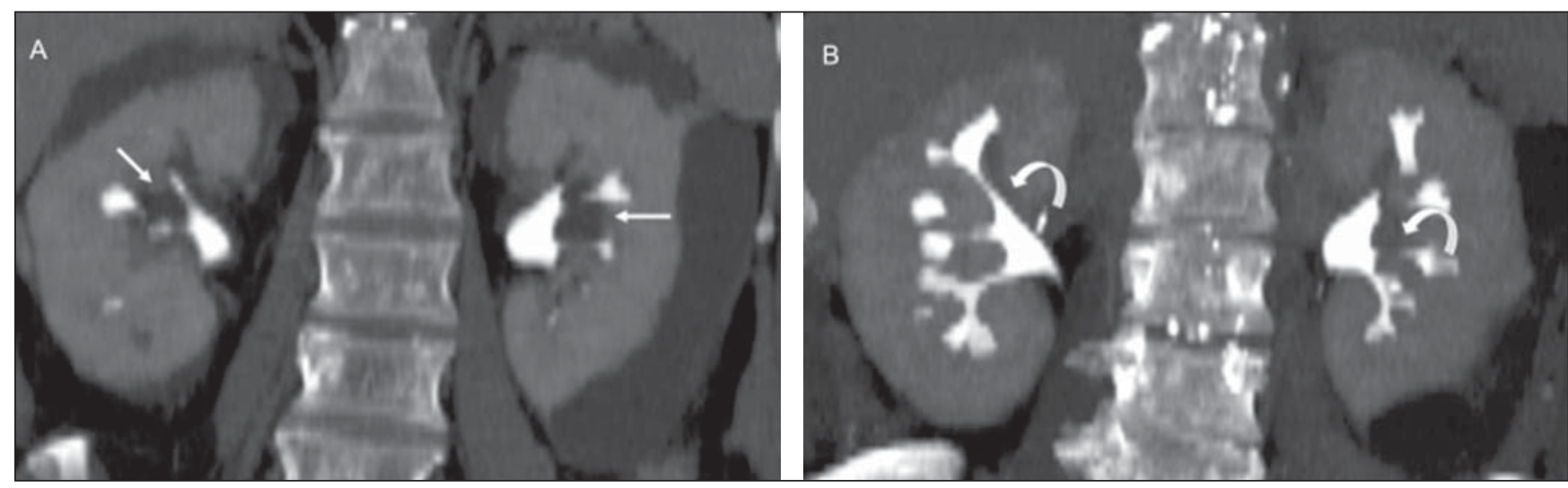

Figura 2. Reformatações MIP no plano coronal de cortes em fase excretora demonstrando pequenas imagens císticas parapiélicas (setas retas em A) determinando discreto alongamento e deformidade de grupos calicinais (setas curvas em B).

ragia intracística, pode haver aumento da atenuação. Pode-se ainda visualizar espessamento das fáscias renais ${ }^{(\mathbf{1})}$ e coleções retroperitoneais cruzando a linha média ao nível do hilo renal ${ }^{(\mathbf{1 , 2})}$.

Na ressonância magnética as lesões são visualizadas como hiperintensas em T2 e hipointensas em T1. Em casos de hemorragia, focos de hipersinal podem ser vistos no interior dos cistos em T1. Observam-se, ainda, hipersinal do córtex e hipossinal relativo da medula em T2. Sugere-se que este padrão de inversão corticomedular, visualizado apenas em $\mathrm{T} 2$, poderia ser devido a edema/congestão renal secundária à obstrução linfática, explicando também a hiperecogenicidade e perda da diferenciação corticomedular na ultrassonografia ${ }^{(1,3)}$.
No diagnóstico diferencial várias possibilidades podem ser aventadas. Abscesso ou urinoma podem ser diferenciados com base na história clínica, por eventual realce ou extravasamento de meio de contraste a partir do sistema coletor ${ }^{(\mathbf{3 , 4})}$. A doença renal policística hereditária apresenta múltiplos cistos acometendo predominantemente o córtex renal, além de mais comumente de- 


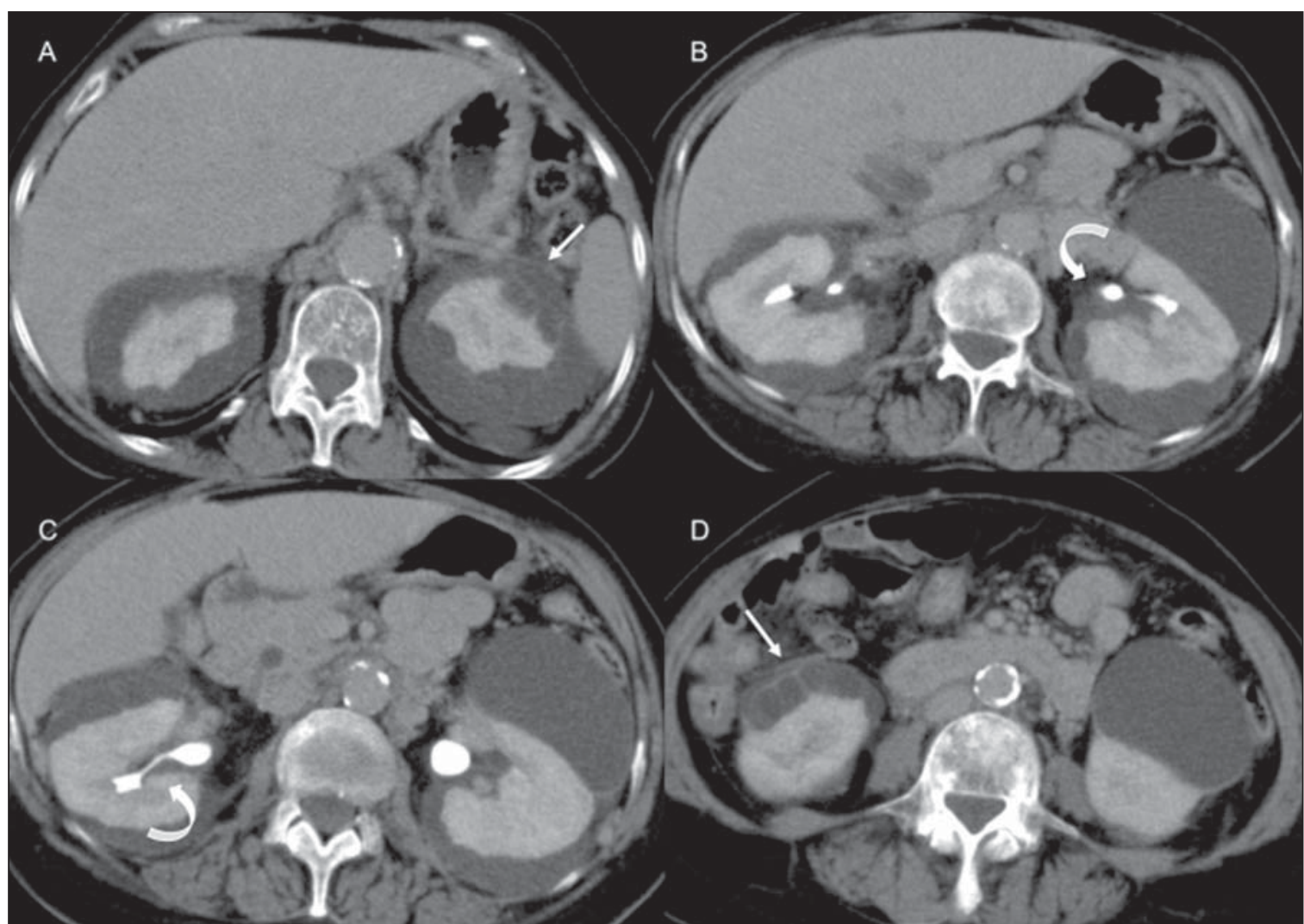

Figura 3. Cortes axiais em fase excretora demonstrando a irregularidade do contorno cortical renal bilateral associada a várias imagens císticas perinéfricas septadas (setas retas em $\mathbf{A}$ e $\mathbf{D}$ ) e parapiélicas com alongamento dos cálices (setas curvas em $\mathbf{B}$ e $\mathbf{C}$ ).

terminar função renal alterada e envolvimento de outros órgãos ${ }^{(\mathbf{1}, 2,4)}$. Linfoma e outras malignidades podem ser diferenciadas por acometerem o parênquima cortical renal, em função da sua atenuação de partes moles e eventual realce pelo contraste ${ }^{(1,2)}$.

Apesar dos aspectos clinicorradiológicos bem definidos, a história natural da doença permanece incerta ${ }^{(2)}$. Há relatos de casos diagnosticados incidentalmente que não apresentaram perda da função renal durante seguimento ${ }^{(\mathbf{1 , 4})}$. Porém, raros casos evoluíram para insuficiência renal crônica. Outra situação importante consiste em sua ocorrência durante a gestação, com deterioração da função renal e hipertensão arterial, assemelhando-se a quadro de préeclâmpsia, com reversão pós-parto.

Outras complicações descritas foram infecção superposta, hemorragia intracís- tica $^{(4)}$, hipertensão arterial grave e prolon$\operatorname{gada}^{(7)}$, uropatia obstrutiva ${ }^{(5)}$ e trombose de veias renais ${ }^{(8)}$.

Considerando que a grande maioria dos pacientes é assintomática, nenhum tratamento é usualmente necessário ${ }^{(\mathbf{1 , 3})}$. Entretanto, em função da possibilidade de uma evolução para insuficiência renal crônica e complicações, acompanhamento clínico e imaginológico deve ser realizado periodicamente $\mathrm{d}^{(\mathbf{4 , 5}, \mathbf{8})}$. Além disso, pacientes femininas em idade reprodutora devem ser orientadas a respeito dos riscos durante uma gestação ${ }^{(4)}$. Para a grande maioria dos pacientes sintomáticos com grandes coleções, drenagem percutânea pode ser utilizada como tratamento conservador, reservando-se a cirurgia para casos recorrentes e coleções complicadas ou incontroláveis $^{(\mathbf{1}, \mathbf{3}-\mathbf{5})}$.

\section{REFERÊNCIAS}

1. Rastogi R, Rastogi V. Computed tomographic scan in the diagnosis of bilateral renal lymphangiectasia. Saudi J Kidney Dis Transpl. 2008;19:976-9.

2. Ramseyer LT. Case 34: renal lymphangiectasia. Radiology. 2001;219:442-4.

3. Mani NB, Sodhi KS, Singh P, et al. Renal lymphangiomatosis: a rare cause of bilateral nephromegaly. Australas Radiol. 2003;47:184-7.

4. Özmen M, Deren Ö, Akata D, et al. Renal lymphangiomatosis during pregnancy: management with percutaneous drainage. Eur Radiol. 2001;11:37-40.

5. Chiu JS, Wu CJ, Sun GH, et al. Obstructive uropathy associated with bilateral renal lymphangiomatosis. Nephrol Dial Transplant. 2004;19:2923.

6. Varela JR, Bargiela A, Requejo I, et al. Bilateral renal lymphangiomatosis: US and CT findings. Eur Radiol. 1998;8:230-1.

7. Lindsey JR. Lymphangiectasia simulating polycystic disease. J Urol. 1970;104:658-62.

8. Riehl J, Schmitt H, Schäfer L, et al. Retroperitoneal lymphangiectasia associated with bilateral renal vein thrombosis. Nephrol Dial Transplant. $1997 ; 12: 1701-3$. 